\title{
Estratégias para reorganização da atenção hospitalar no Paraná para enfrentamento da Covid-19
}

\author{
Strategies to reorganize hospital care in Paraná in \\ confronting Covid-19
}

Andreia Clerice da Silva Barbosa ${ }^{1}$, Jéssica Oliveira de Lima², Maria Goretti David Lopes³, Carmen Cristina Moura dos Santos ${ }^{4}$

\begin{abstract}
1. ORCID: https://orcid.org/0000-0001-6548-2133. Enfermeira. Universidade Federal do Paraná (UFPR). Secretaria de Estado da Saúde do Paraná (Curitiba, Paraná, Brasil). E-mail: andreia.clerice@sesa.pr.gov.br.

2. ORCID: https://orcid.org/0000-0003-1264-2193. Enfermeira especialista em Saúde da Família (Faculdades Pequeno Príncipe - 2016) e em Gestão da Saúde (Universidade Federal do Paraná - 2019). Secretaria de Estado da Saúde do Paraná (Curitiba, Paraná, Brasil). E-mail: jessica. oliveira@sesa.pr.gov.br.

3. ORCID: https://orcid.org/0000-0001-7473-6342. Enfermeira. Diretora de Atenção e Vigilância em Saúde da Secretaria de Estado da Saúde do Paraná (Curitiba, Paraná, Brasil).

4. ORCID: https://orcid.org/0000-0003-3162-8346. Enfermeira. Coordenadora da Organização da Rede de Cuidados em Saúde da Secretaria de Estado da Saúde do Paraná (Curitiba, Paraná, Brasil).
\end{abstract}

CONTATO: Andreia Clerice da Silva Barbosa | Rua Piquiri, n 170, Rebouças, Curitiba. Telefone: (41) 99148-7105 | E-mail: deia_clerice@yahoo.com.br | andreia.clerice@sesa.pr.gov.br

RESUMO O enfrentamento da Covid-19 exige rápida reorganização da Atenção Hospitalar para garantir a retaguarda de atendimento aos casos de maior gravidade. Apesar das evidências apontarem que na maioria dos infectados a doença provoca quadros gripais leves, uma parcela destes exige manejo e tratamento clínico em unidades de maior complexidade tecnológica. Para garantir acesso universal ao tratamento hospitalar, foi necessário preparar a Rede de Atenção à Saúde, com foco na retaguarda de leitos intensivos, a fim de suprir a demanda adicional projetada. Este relato de experiência apresenta estratégias da Secretaria de Estado da Saúde do Paraná relativas ao aumento do aporte de leitos em Unidades de Terapia Intensiva, ações para reorganização 
do processo de trabalho nos hospitais, aporte de equipamentos de proteção individual e investimentos emergenciais em estruturas físicas com potencial capacidade de operação. Desafios cotidianos demandam inovação com foco na saúde dos trabalhadores e usuários do sistema de saúde.

DESCRITORES: Pandemias. Coronavírus. Hospitais. Unidades de Terapia Intensiva.

ABSTRACT In facing Covid-19, hospital care needs to be quickly reorganized to safeguard and guarantee the treatment of the more serious cases. Even though the evidence shows that the majority of people infected with the disease show light symptoms of the flu, a portion of cases requires clinical treatment and management in hospital units with more complex technology. To make sure that everyone received equal access to hospital care, it was necessary to form a Health Care Network, with the focus on keeping beds in intensive care units available to meet the increasing projected demands. This experience report presents strategies from the State Secretary of Health of Paraná in relation to the increased contribution of beds for ICUs, actions to reorganize the work flow in hospitals, the contribution of individual protective outfits, and emergency investments to expand the hospitals' physical structures, enabling a greater capacity for operation. Daily challenges demand innovation to benefit the health of both the health care workers and health system users.

DESCRIPTORS: Pandemics. Coronavirus. Hospitals. Intensive Care Units.

\section{INTRODUÇÃO}

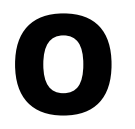

mundo vive um momento de apreensão gerada por uma nova doença do trato respiratório, denominada Covid-19, causada pelo vírus SARS-CoV-2. Grande parte dos infectados apresenta manifestações clínicas brandas, com sintomas característicos de síndrome gripal. Entretanto, em 14\% dos casos pode desencadear insuficiência respiratória grave e em $5 \%$ dos pacientes o manejo clínico poderá exigir a intubação orotraqueal e ventilação mecânica1. Esta situação demanda a organização da atenção hospitalar, e consequente suprimento e organização de leitos de retaguarda ${ }^{2-3}$. Nos quadros graves, terapias e monitoramento de alta densidade deverão ser empregados, precocemente, preenchendo os critérios de gravidade para ocupação de leitos de Unidade de 
Terapia Intensiva (UTI)².

Diante desse cenário, respostas rápidas para o enfrentamento da pandemia emergente no Brasil foram primordiais, a fim de evitar o saturamento do sistema de saúde. A Rede de Urgência e Emergência (RUE) e a Atenção Hospitalar foram o enfoque das ações iniciais, destacando-se: ampliação da capacidade das unidades; construção de novas unidades hospitalares e organização de hospitais de campanha; contratação de leitos em hospitais privados ou do setor suplementar; aquisição de insumos e equipamentos; provisão de recursos humanos para atender às especificidades da doença; e regulação estratégica dos leitos disponíveis, com priorização da demanda às vagas adequadas para cada caso $^{2}$.

Nesse contexto, este relato de experiência tem por objetivo evidenciar a disponibilidade de leitos de UTI, destinados à Covid-19, fora do período e no período pandêmico, com destaque às modificações demandadas a partir do surgimento da emergência em saúde pública no Estado do Paraná.

\section{MÉTODO}

Trata-se de relato de experiência que apresenta as estratégias adotadas pela Secretaria de Estado da Saúde do Paraná (SESA) para o enfrentamento da pandemia por Covid-19, em relação à organização da atenção hospitalar. Para o levantamento das informações relacionadas ao número de leitos intensivos, exclusivos ou não, para a COVID-19, foram consultados os dados do Cadastro Nacional de Estabelecimentos de Saúde (CNES) e do DATASUS.

Para fins de comparação entre os leitos disponíveis e os contratados frente à pandemia, utilizou-se como referência os dados do primeiro semestre de 2019, em comparação ao mesmo período de 2020. A estimativa populacional foi obtida a partir das informações do Instituto Brasileiro de Geografia e Estatística (IBGE), tendo por base o ano de 2019. 
Por tratar-se de bancos de dados de acesso público, esta publicação não foi submetida à apreciação por Comitê de Ética em Pesquisa, conforme orienta a Resolução do Conselho Nacional de Saúde n 510, de 7 de abril de 2016.

\section{RESULTADOS E DISCUSSÃO}

No Estado do Paraná, as ações para o enfrentamento da pandemia iniciaram-se no mês de fevereiro de 2020, com a ativação do Centro de Operações em Emergências (COE), por meio das Resoluções SESA PR $n^{\circ} 126 / 2020^{4}$ e $n^{\circ}$ 317/20205, dando início às discussões para reorganização da Rede de Atenção à Saúde (RAS) estadual e, em especial, da atenção hospitalar, foco desse relato de experiência.

Em abril de 2020, o Plano de Contingência ${ }^{6}$ estadual foi elaborado, em decorrência das discussões realizadas pelos membros do $\mathrm{COE}$, com o intuito de orientar os componentes da RAS na rápida e efetiva resposta para enfrentamento da Covid-19, articulando-se com as vigilâncias em saúde, órgãos de classe e demais entidades da sociedade organizada. Em junho de 2020 o documento foi atualizado, elevando a organização de resposta para o nível 3 de execução7.

Ao acompanhar a evolução da doença em outros países, ficou evidente a necessidade de resposta rápida e eficiente para mitigação dos agravos decorrentes do aumento nas taxas de contaminação, exigindo a organização e ampliação na oferta de leitos hospitalares, principalmente no que se refere aos leitos intensivos, aprimorando os serviços existentes e prevendo os recursos que serão necessários para o devido enfrentamento da pandemiå .

As UTI são áreas destinadas à internação e cuidados ao paciente grave, o qual requer o uso de tecnologia, monitorização, terapia e insumos específicos, além de profissionais especializados ${ }^{9}$.

No Paraná, a soma dos leitos existentes e com atendimento exclusivo ao Sistema Único de Saúde (SUS), de UTI adulto Tipo I, Tipo II, Tipo III, Coronaria- 
na Tipo II, Tipo III, UTI de Queimados e Unidade de Cuidados Intermediários Adulto, bem como a soma dos leitos de UTI Pediátrica Tipo I, Tipo II, Tipo III e Unidade de Cuidados Intermediários Pediátrico, no período de janeiro a junho de 2019 e 2020, são apresentados na Tabela 1.

Tabela 1. Distribuição da comparação dos leitos intensivos para internação de adulto e pediátrico em 2019 e 2020 no Estado do Paraná.

\begin{tabular}{|c|c|c|c|c|c|}
\hline \multirow{2}{*}{$\begin{array}{l}\text { Período } \\
\text { ADULTO }\end{array}$} & \multicolumn{2}{|c|}{ Jan-Jun 2019} & \multicolumn{2}{|c|}{ Jan-Jun 2020} & \multirow{2}{*}{$\begin{array}{c}\text { Variação } \\
\text { 2019-2020 } \\
(\%)\end{array}$} \\
\hline & Existentes & sus & Existentes & sus & \\
\hline Janeiro & 2.169 & 1.247 & 2.244 & 1.315 & 3,4 \\
\hline Fevereiro & 2.188 & 1.247 & 2.258 & 1.315 & 3,1 \\
\hline Março & 2.192 & 1.247 & 2.155 & 1.277 & $-1,6$ \\
\hline Abril & 2.182 & 1.247 & 2.292 & 1.279 & 5,0 \\
\hline Maio & 2.182 & 1.247 & 2.379 & 1.273 & 9,0 \\
\hline Junho & 2.177 & 1.247 & 2.280 & 1.279 & 4,7 \\
\hline $\begin{array}{l}\text { TOTAL } \\
\text { (média) }\end{array}$ & 2.181 & 1.247 & 2.268 & 1.289 & 4,0 \\
\hline PEDIÁTRICO & Existentes & sus & Existentes & sus & Variação \\
\hline Janeiro & 256 & 176 & 254 & 178 & $-0,7$ \\
\hline Fevereiro & 256 & 176 & 254 & 178 & $-0,7$ \\
\hline Março & 259 & 176 & 257 & 178 & $-0,7$ \\
\hline Abril & 259 & 176 & 253 & 178 & $-2,3$ \\
\hline Maio & 258 & 176 & 252 & 178 & $-2,3$ \\
\hline Junho & 257 & 176 & 252 & 178 & $-1,9$ \\
\hline $\begin{array}{l}\text { TOTAL } \\
\text { (média) }\end{array}$ & 257 & 176 & 253 & 178 & $-1,5$ \\
\hline
\end{tabular}

Fonte: Cadastro Nacional de Estabelecimentos de Saúde (CNES), 2020.

De acordo com a modalidade de leitos intensivos para adultos, notou-se constância no Estado do Paraná em 2019, com incremento médio de 4,0\% em 
2020, e decréscimo da oferta no mês de março do mesmo ano. Nos demais meses notou-se uma variação positiva, com destaque para maio de 2020, que apresentou aumento de 9,0\% no número de leitos, quando comparado ao mesmo período de 2019.

Ao considerar a relação média de leitos com atendimento exclusivo ao SUS em 2019 e a estimativa populacional do IBGE, o Paraná apresentava 1,09 leitos de UTI adulto para cada 10 mil habitantes. Ao considerar o total de leitos de UTI em 2020, esse número se eleva para 1,90 leitos. Evidenciou-se, portanto, que anteriormente ao período pandêmico havia adequado aporte de leitos intensivos adultos, segundo recomendação da OMS e MS, a qual determina entre 1 e 3 leitos de UTI a cada 10 mil habitantes ${ }^{10}$.

Quanto aos leitos pediátricos (Tabela 1), observou-se um decréscimo médio de 1,5\% entre 2019 e 2020, com maior redução de leitos não SUS.

Por meio da publicação da Portaria n 414 de 18 de março de 2020, do MS, foi autorizada a habilitação de leitos exclusivos para Covid-19, sendo criada no CNES a descrição "UTI II adulto Covid-19" e "UTI II pediátrica Covid-19" para sua identificação ${ }^{11}$. Já a Portaria n 1.206, de 12 de maio de 2020, do MS, habilitou aporte significativo de leitos intensivos adulto e pediátricos exclusivos no Paraná, e também estabeleceu o repasse de recurso financeiro por meio do Bloco de Manutenção das Ações e Serviços Públicos de Saúde, a ser disponibilizado ao Estado e municípios, para manutenção desses pelo período de 90 dias $^{12}$.

Na Tabela 2 são apresentados os leitos habilitados no primeiro semestre de 2020, por Macrorregião, na qual observou-se importante e gradativo aporte de leitos para população adulta, o que difere dos leitos pediátricos, que apresentam número constante. Essa diferença ocorre devido à demanda desigual de internamento em UTI, conforme a faixa etária dos pacientes acometidos. As evidências reportam para manifestação mais tênue da doença em crianças e adolescentes, podendo ser assintomáticos ou apresentarem um quadro da Covid-19 sem gravidade ${ }^{13}$. 
Tabela 2. Distribuição dos leitos intensivos para paciente adulto e pediátrico - exclusivos Covid-19 - de acordo com a macrorregião no Estado do Paraná no ano de 2020.

\begin{tabular}{ccc}
\hline Macrorregião & $\begin{array}{c}\text { Adulto } \\
\text { Jan-Jun }\end{array}$ & $\begin{array}{c}\text { Pediátrico } \\
\text { Jan-Jun }\end{array}$ \\
\hline Leste & 406 & 21 \\
Oeste & 104 & 2 \\
Noroeste & 112 & 11 \\
Norte & 163 & 3 \\
Total & $\mathbf{7 8 5}$ & 37 \\
\hline
\end{tabular}

Fonte: Informe Epidemiológico Coronavírus (Covid-19) - SESA-PR, 2020.

Considerando as diferenças populacionais e a dinâmica da pandemia no Estado, houve também habilitação diferenciada de leitos por macrorregião, com expressivo aporte para a Macrorregião Leste do Estado, a qual possuía, aproximadamente, $51 \%$ do total de 785 leitos de UTI adulto no mês de junho. Essa proporção pode ser compreendida ao analisar a Tabela 3, que apresenta a estimativa de população de cada macrorregião, sendo a Leste responsável por $48,9 \%$ do total de habitantes do Estado.

Tabela 3 - Distribuição da população residente segundo Macrorregião de saúde do Estado do Paraná, no ano de 2019.

\begin{tabular}{|c|c|c|c|c|}
\hline Macrorregião & $\begin{array}{c}\mathbf{N}^{\circ} \text { de } \\
\text { Habitantes }\end{array}$ & $\mathbf{N}^{\circ}$ de & Municípios & Porcentagem (\%) \\
\hline Leste & 5.586 .569 & & 93 & 48,9 \\
\hline Oeste & 1.978 .824 & & 94 & 17,3 \\
\hline Noroeste & 1.879 .867 & & 115 & 16,4 \\
\hline Norte & 1.988 .697 & & 97 & 17,4 \\
\hline Total & 11.433.957 & & 399 & 100,0 \\
\hline
\end{tabular}

Fonte: Estimativa Populacional por Macrorregião período 2019 - DATASUS, 2020. 
As recomendações internacionais apontam para a necessidade de reorganização da atenção hospitalar, e da definição de serviços de referência destinados ao tratamento específico da Covid-19. Demanda-se, igualmente, um mecanismo de gestão de leitos, com dados orientativos, tais como taxa de ocupação e disponibilidade dos leitos exclusivos, em tempo real, imprescindíveis para gestão eficiente do sistema de saúde nesse nível de atenção ${ }^{14}$.

No Paraná, a gestão dos leitos intensivos habilitados, exclusivamente, para atendimento a Covid-19, é efetuada por uma central de regulação estadual. Diariamente são publicadas informações em relação ao número de leitos disponíveis, e sobre a taxa de ocupação por Macrorregião do Estado, que pode ser consultada no informe epidemiológico da SESA-PR, como exemplo do mês de julho de 2020, apresentado na Tabela 4.

Tabela 4. Distribuição dos leitos intensivos SUS exclusivos para pacientes suspeitos ou confirmados Covid-19 - Por Macrorregião do Estado do Paraná no mês 07/2020.

\begin{tabular}{c|ccc|ccc}
\cline { 2 - 7 } Macrorregião & Existentes & Ocupados & $\begin{array}{c}\text { Tx de } \\
\text { ocupação }\end{array}$ & Existentes & Ocupados & $\begin{array}{c}\text { Txde } \\
\text { ocupação }\end{array}$ \\
\hline Leste & 513 & 462 & $90 \%$ & 21 & 9 & $43 \%$ \\
Oeste & 143 & 98 & $69 \%$ & 2 & 2 & $100 \%$ \\
Noroeste & 132 & 81 & $61 \%$ & 11 & 1 & $9 \%$ \\
Norte & 173 & 97 & $56 \%$ & 3 & 1 & $33 \%$ \\
Total & $\mathbf{9 6 1}$ & $\mathbf{7 3 8}$ & $\mathbf{7 7 \%}$ & $\mathbf{3 7}$ & $\mathbf{1 3}$ & $\mathbf{3 5 \%}$ \\
\hline
\end{tabular}

Fonte: Informe Epidemiológico Coronavírus (Covid-19) - SESA-PR, em 17/07/2020.

A análise da tabela mostra alta taxa de ocupação de leitos na Macrorregião Leste, sede da capital paranaense e área de maior densidade populacional. Sendo necessária a gestão adequada dos leitos intensivos, por meio da atuação da central de regulação organizada em rede, fundamental para otimizar a oferta de vagas. 
No período anterior à pandemia, o tempo médio de permanência de um paciente em leito intensivo de hospital público era de, aproximadamente, 6,5 dias. Com as características diferenciadas da enfermidade causada pelo SARS-CoV-2, a média de permanência saltou para 14 dias, representando um desafio para os gestores ${ }^{10}$ por representar menor rotatividade.

Ao encerrar o primeiro semestre de 2020 com 785 leitos adultos exclusivos Covid-19 (Tabela 2), habilitados para o enfrentamento da pandemia no Paraná, observou-se aporte adicional de $36 \%$ de leitos intensivos quando comparado ao mesmo período em 2019. Entretanto, ainda se vivencia o aumento do número de casos confirmados e das taxas de ocupação de leitos intensivos, e novas habilitações são necessárias a fim de garantir a retaguarda de atendimento.

Evidências garantem que em períodos pandêmicos a necessidade de aumento de novos leitos intensivos pode se elevar em cerca de $200 \%$ em relação à capacidade instalada. Fato que reitera a demanda por reestruturar os serviços hospitalares, concomitante a outras situações de saúde que não deixaram de existir, constituindo desafio maior ${ }^{15}$.

Outras estratégias para a reorganização da atenção hospitalar foram empregadas pela SESA-PR nesse período, e são de caráter permanente. Estruturas hospitalares que se encontravam em construção ou reforma antes da pandemia foram o foco de atuação da gestão estadual, com o objetivo de utilizá-las como referência regional de atendimento. Investimentos em hospitais regionais, como nos municípios de Ivaiporã, Guarapuava e Telêmaco Borba, foram realizados. Essas estruturas tiveram seus processos de reforma e construção acelerados para atender à demanda crescente, sendo que as previsões anteriores para entrega eram para dezembro de 2020. Ativações de instalações novas se somaram ao número de leitos já existentes em todo o Estado ${ }^{16}$.

Novos leitos intensivos, que estavam em processo de finalização antes da pandemia, também foram habilitados, como a exemplo, no Hospital Regional do Norte Pioneiro, em Santo Antônio da Platina, que teve dez leitos de UTI ha- 
bilitados inicialmente para atendimento à Covid-19, os quais permanecerão ativos após a emergência em saúde pública ${ }^{17}$ assim como as demais estruturas instaladas, agregando-se à RAS nas diversas macrorregiões paranaenses.

No município de Curitiba, utilizou-se como estratégia para a organização da atenção hospitalar o uso de estruturas físicas hospitalares desativadas da iniciativa privada. Por intermédio da parceria entre a SESA e os responsáveis de dois complexos hospitalares privados da capital, foi possível disponibilizar aporte temporário de leitos intensivos e clínicos para enfrentamento à pandemia ${ }^{18-19}$.

Considerando a emergência de saúde pública, a Resolução SESA-PR n 338, de março de 2020 recomendou a suspensão temporária de procedimentos cirúrgicos eletivos, com garantia de reagendamento posterior ao quadro pandêmico vigente. Excetuaram-se, dessa recomendação, os casos com potencial de prejuízo funcional ou sequela definitiva ${ }^{20}$.

A recomendação em relação à suspensão temporária objetivou diminuir a circulação de pacientes em ambientes hospitalares de forma programada, sem caráter de emergência. Além disso, levou em consideração a preservação da disponibilidade de equipamentos de proteção individual e medicamentos sedativos utilizados em intubações orotraqueais. Os profissionais de saúde dos setores eletivos foram contabilizados como mãos de obra auxiliar à demanda aumentada nesse nível de atenção ${ }^{20}$.

As comunidades científicas intensivas e gestoras do SUS, em âmbito federal, também entraram em consenso em relação às recomendações citadas no parágrafo anterior. O cancelamento de procedimentos e cirurgias eletivas garante aporte de leitos intensivos significativo, que antes serviriam de retaguarda para possíveis complicações desses procedimentos, além do uso racional de insumos medicamentosos, diagnósticos e de equipamentos de proteção individual (EPI) $)^{1-10}$. 


\section{CONSIDERAÇÕES FINAIS}

Considerando o cenário inédito e o desafio imposto pela pandemia por Covid-19 no Estado, as ações de enfrentamento da SESA-PR na atenção hospitalar tiveram por objetivo garantir à população direito de acesso a leitos intensivos no caso de agravamento da doença, e uma assistência à saúde de qualidade nesse nível de atenção. As estratégias da gestão, para ampliação do número de leitos intensivos, reorganização dos processos de trabalho nos hospitais, e a parceria público-privada, permitiram aos pacientes com agravamento do quadro clínico pelo SARS-CoV-2 melhores condições de tratamento e prognóstico.

Entretanto, a pandemia permanece em curso, demandando a adoção de estratégias que superam a habilitação de novos leitos, exigindo a colaboração da sociedade para mitigar o ciclo de transmissão da doença, e o comprometimento dos gestores nas pactuações e gestão assertiva dos recursos. Vale lembrar que ações para proteção das equipes de saúde, como o aporte adequado de equipamentos de proteção individual, além da oferta suficiente de insumos e medicamentos são relevantes neste contexto assistencial.

\section{AGRADECIMENTOS}

Agradecemos à Elaine Drehmer de Almeida Cruz, apoiadora pela OPAS junto à SESA-PR, pelas contribuições ao texto.

\section{REFERÊNCIAS}

1. Conselho Nacional dos Secretários de Saúde (CONASS). COVID-19: Guia orientador para o enfrentamento da pandemia na Rede de Atenção à Saúde. Brasília: CONASS; 2020. [citado em 2020 Mai 20]. Disponível em: https://www.conasems.org. br/wp-content/uploads/2020/05/Instrumento-Orientador-Conass-Conasems-VERS\%C3\%83O-FINAL-3.pdf.

2. Brasil. Ministério da Saúde. Orientações para o manejo de pacientes com COVID-19. Coronavírus: COVID-19. Brasília; 2020. [citado em 2020 Jun 15]. Disponível em: https://portalarquivos.saude.gov.br/images/pdf/2020/June/18/Covid19Orientac--o--esManejoPacientes.pdf.

3. Lana RM, Coelho FC, Gomes MFC, Cruz OG, Bastos LS, Villela DAM et al. Emergência do novo coronavírus (SARS-CoV-2) e o papel de uma vigilância nacional em saúde oportuna e efetiva. Perspectivas - Caderno Saúde Pública 36 (3) 13 Mar 2020. [citado em 2020 Jun 12]. Disponível em: https://scielosp.org/article/csp/2020.v36n3/e00019620/pt/. 
4. Paraná. Secretaria de Saúde do Paraná. Resolução n¹26/2020. Ativa o Centro de Operações em Emergências - COE para o enfrentamento do novo Coronavírus (2019-nCoV). Curitiba; 2020. [citado em 2020 Mai 20]. Disponível em: http://www. saude.pr.gov.br/sites/default/arquivos_restritos/files/documento/2020-04/126_20.pdf.

5. Paraná. Secretaria de Saúde do Paraná. Resolução n³17/2020. Ampliação da composição e atribuições do Centro de Operações de Emergências em Saúde Pública - COE da Secretaria de Estado do Paraná para o enfrentamento do novo coronavírus (COVID-19), febre amarela, dengue e outros agravos que exijam respostas rápidas, no âmbito do Sistema Único de Saúde. Curitiba; 2020. [citado em 2020 Mai 2]. Disponível em: http://www.saude.pr.gov.br/sites/default/arquivos restritos/files/documento/2020-04/317_20.pdf.

6. Paraná. Secretaria de Saúde do Paraná. Centro de Operações em Emergências. Plano de Contingência do Paraná COVID-19. Curitiba; 2020. [citado em 2020 Mai 28]. Disponível em: http://www.saude.pr.gov.br/sites/default/arquivos_ restritos/files/documento/2020-04/planocovid19final.pdf.

7. Paraná. Secretaria de Saúde do Paraná. Centro de Operações em Emergências. Plano de Contingência do Paraná COVID-19. Nível 3 - Execução. Curitiba; 2020. [citado em 2020 Jul 1]. Disponível em: http://www.saude.pr.gov.br/sites/default/ arquivos_restritos/files/documento/2020-06/plano_de_contingencia_do_parana_covid_19_nivel_3_atualizado.pdf.

8. Rache B, Rocha R, Nunes L, Spinola P, Malik AM, Massuda A. Necessidades de Infraestrutura do SUS em Preparo ao COVID-19: Leitos de UTI, Respiradores e Ocupação Hospitalar. Nota Técnica n 3. São Paulo: IEPS; 2020. [citado em 2020 Jun 20]. Disponível em: http://www.epsjv.fiocruz.br/sites/default/files/files/NT3\%20vFinal.pdf.

9. Brasil. Agência Nacional de Vigilância Sanitária. Resolução nº 7 de 24 de fevereiro de 2010. Dispõe sobre os requisitos mínimos para funcionamento de Unidades de Terapia Intensiva e dá outras providências. [citado em 2020 Jul 10]. Disponível em: http://bvsms.saude.gov.br/bvs/saudelegis/anvisa/2010/res0007_24_02_2010.html.

10. Associação de Medicina Intensiva Brasileira (AMIB). Comunicado da AMIB sobre o avanço do COVID-19 e a necessidade de leitos em UTIs no futuro. [citado em 2020 Jul 12]. Disponível em: http://www.somiti.org.br/arquivos/site/comunicacao/ noticias/2020/covid-19/comunicado-da-amib-sobre-o-avanco-do-covid-19-e-a-necessidade-de-leitos-em-utis-no-futuro. pdf.

11. Brasil. Portaria $n^{\circ}$ 414, de 18 de março de 2020. Autoriza a habilitação de leitos de Unidade de Terapia Intensiva Adulto e Pediátrico, para atendimento exclusivo dos pacientes COVID-19. 2020 Mar 20. Edição 55. Seção 1. [citado em 2020 Mai 12]. Disponível em: http://www.in.gov.br/web/dou/-/portaria-n-414-de-18-de-marco-de-2020-249025222.

12. Brasil. Portaria $n^{\circ} 1206$, de 12 de maio de 2020. Habilita leitos da Unidade de Terapia Intensiva - UTI Adulto Tipo II - COVID-19 e UTI Pediátrico Tipo II - COVID-19 e estabelece recurso do Bloco de Manutenção das Ações e Serviços Públicos de Saúde - Grupo Coronavírus (COVID 19), a ser disponibilizado ao Estado do Paraná e Municípios. 2020 Mai 19. Edição 94. Seção 1. [citado em 2020 Mai 27]. Disponível em: http://www.in.gov.br/web/dou/-/portaria-n-1.206-de-12-maiode-2020-*-257392320.

13. Brasil. Ministério da Saúde. Secretaria de Atenção Especializada à Saúde. Departamento de Atenção Hospitalar, Domiciliar e de Urgência. Protocolo de manejo clínico da Covid-19 na Atenção Especializada [Internet]. Ministério da Saúde, Secretaria de Atenção Especializada à Saúde, Departamento de Atenção Hospitalar, Domiciliar e de Urgência. 1. ed. rev. Brasília; 2020. [citado em 2020 Jun 14]. Disponível em: http://bvsms.saude.gov.br/bvs/publicacoes/manejo_clinico_covid-19_ atencao_especializada.pdf.

14. Organização Pan Americana de Saúde (OPAS). Reorganização e Expansão Progressiva dos Serviços de Saúde para Resposta à Pandemia de COVID-19. OPAS/OMS. 2020 Mar. [citado em 2020 Jul 12]. Disponível em: https://iris.paho.org/ bitstream/handle/10665.2/52068/OPASBRACOVID1920030_por.pdf?sequence=3\&isAllowed=y.

15. Hick JL, Einav S, Hanfling D, Kissoon N, Dichter JR, Devereaux AV et al. Surge capacity principles: care of the critically ill and injured during pandemics and disasters: CHEST consensus statement. Chest, 146(4), e1S-e16S, 2014.

16. Paraná. Secretaria de Saúde do Paraná. Governo do Paraná amplia estrutura hospitalar contra a COVID-19. 2020 Abr. [citado em 2020 Jul 12]. Disponível em: http://www.saude.pr.gov.br/Noticia/Governo-do-Parana-amplia-estrutura-hospitalarcontra-Covid-19. Acesso eem 12/07/2020.

17. Paraná. Secretaria de Saúde do Paraná. UTI do Hospital Regional do Norte Pioneiro entra em funcionamento. [citado em 2020 Jul 12]. Disponível em: http://www.saude.pr.gov.br/Noticia/UTI-do-Hospital-Regional-do-Norte-Pioneiro-entra-emfuncionamento.

18. Curitiba. Secretaria de Saúde de Curitiba. Curitiba abre mais leitos e chega a 646 exclusivos para COVID-19. [citado em 2020 Jul 12]. Disponível em: http://www.saude.curitiba.pr.gov.br/noticias/23-noticias-principal/1620-curitiba-abre-maisleitos-e-chega-a-646-exclusivos-para-covid-19.html.

19. Curitiba. Secretaria de Saúde de Curitiba. Parceria garante 140 novos leitos exclusivos para COVID-19 em Curitiba. 
[citado em 2020 Jul 14]. Disponível em: http://www.saude.curitiba.pr.gov.br/noticias/1551-parceria-garante-140-novosleitos-exclusivos-para-covid-19-em-curitiba.html.

20. Paraná. Secretaria de Saúde do Paraná. Resolução n 338/2020. Implementação de medidas de enfrentamento da emergência em saúde pública de importância nacional e internacional decorrente do Coronavírus - COVID-2019. [citado em 2020 Jun 14]. Disponível em: http://www.saude.pr.gov.br/sites/default/arquivos_restritos/files/documento/2020-04/338_20.pdf.

RECEBIDO: 31/07/2020

ACEITO: 13/10/2020 